Western University

Scholarship@Western

8-1-2019

Probing mid-infrared plasmon resonances in extended radial fractal structures.

Gregory Q Wallace

Danielle M McRae

François Lagugné-Labarthet

flagugne@uwo.ca

Follow this and additional works at: https://ir.lib.uwo.ca/chempub

Part of the Chemistry Commons

Citation of this paper:

Wallace, Gregory Q; McRae, Danielle M; and Lagugné-Labarthet, François, "Probing mid-infrared plasmon resonances in extended radial fractal structures." (2019). Chemistry Publications. 207.

https://ir.lib.uwo.ca/chempub/207 


\title{
Probing Mid-Infrared Plasmon Resonances in Extended Radial Fractal Structures
}

\author{
Gregory Q. Wallace, ${ }^{1}$ Danielle M. McRae, ${ }^{1}$ François Lagugné- \\ LABARTHET $^{1, *}$ \\ ${ }^{1}$ The University of Western Ontario (Western University), Department of Chemistry, 1151 Richmond Street, London, Ontario, Canada, N6A5B7.
}

*Corresponding author: flagugne@uwo.ca

Received XX Month XXXX; revised XX Month, XXXX; accepted XX Month XXXX; posted XX Month XXXX (Doc. ID XXXXX); published XX Month XXXX

\begin{abstract}
Infrared antennas made of metallic nanostructures are widely tunable from the near- to the far-infrared range. They can be utilized for a variety of applications such as light harvesting, photonic filters, and their structural linear or circular anisotropy can be exploited to further enhance the sensitivity of spectroscopic measurements. Here, gold dendritic fractal structures that were optimized to exhibit multiple resonances in the midinfrared range were characterized using a scattering-type scanning near-field optical infrared microscope. The spatially resolved infrared maps associated with the individual modes serve as a basis to understand the mode evolution between each fractal generation.
\end{abstract}

http://dx.doi.org/10.1364/OL.99.099999

Metasurfaces have shown their potential for a variety of applications ranging from the terahertz to the UV-Visible spectral ranges as filters, photovoltaic cells, or plasmon-mediated catalysis [1-3]. Surface-enhanced Raman scattering (SERS), infrared absorption (SEIRA) and fluorescence [4-6], also rely on nanoscale regions where the electromagnetic (EM) field is locally enhanced in the near-field of the structure. The linear anisotropy of such antennas, as well as the structural chirality, are additional parameters that can be tuned, thus exploiting the differential response of a metasurface when subjected to an orthogonal set of linearly or circularly polarized fields [7]. Importantly, the distribution, the anisotropy and the density of hot-spots, i.e. spatial positions where the EM field is locally enhanced are also important factors to performing differential surface chemistry using different wavelengths or polarizations to trigger specific surface reactions on a given structure [8]. In this context, fractal structures are of interest because they exhibit a high density of local hot-spots that can be predicted from the fractal geometry [9]. Furthermore, they present multiple resonances that can be exploited individually. It is therefore of interest to know the spatial distribution of these hotspots associated with a given resonance to turn them "on" or "off" for specific surface reactions, and to possibly predict the resonances associated with new fractal generations.

In simple structures, the position of the localized area of an enhanced EM field can be determined intuitively, as in the cases of adjacent nanoparticles, bow-tie assemblies, rod-like structures or a nanoparticle on a metallic mirror. In such examples, the EM field is generally confined in the nanometer-sized gap(s) between the adjacent structures or at the extremities of the rod structures [1012]. However, for more complex structures and assemblies that show multiple resonances and polarization dependence, numerical calculations are critical tools to assess the spatial properties of the resonances and to determine the ideal irradiation conditions [13]. Calculations involving fractal structures are more tedious to conduct because the different length scales inherent to fractal structures need a small lattice calculation parameter over large surfaces, thus requiring costly computation time to converge.

Therefore, the possibility to experimentally map the modes over a complex fractal structure appears as an important advantage to correlate the appearance of new modes or hybridization for each new fractal generation. For mid-IR resonances, scattering-type scanning near-field optical microscopy (s-SNOM) has shown remarkable results to yield highly spatially resolved direct mapping of infrared modes through the simultaneous measurement of the near-field amplitude and phase images. The interpretation of the sSNOM maps for the particular case of extended 2D structures is complex and cannot be simply related to a specific near-field component [14] but is rather a weighted sum of the individual $E_{\mathrm{x}}$, $E_{\mathrm{y}}$ and $E_{\mathrm{z}}$ fields at a selected point [15].

In this work, we image the mid-IR resonances supported by a series of gold dendritic fractals inscribed by electron-beam lithography on $\mathrm{CaF}_{2}$ windows. The full description of their fabrication can be found somewhere else [16]. The Caylee tree-type fractal structures that have a dendrimer-like geometry exhibit a series of resonances that span the near- to far-IR depending on the size of the individual building blocks and the generation order [7, 16 , 17]. In particular, we focus on five-branched second-order 
generation (5BG2, Fig. 1A) and three-branched fifth-order generation (3BG5, Fig. 1B) dendritic fractals. The generation number refers to the number of iterations of the fractal. These fractals were selected as they present important design features namely: i) a large number of inner branches; ii) a high number of generations; iii) a radial-type geometry that supports the possibility of hybridization between fractal generations.
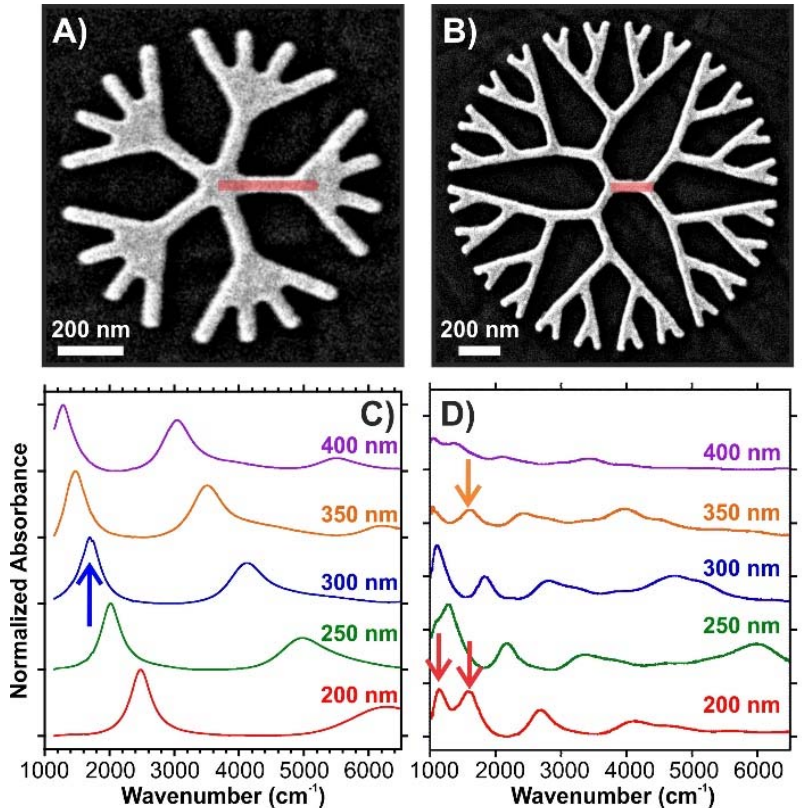

Fig. 1. SEM images of A) 5BG2 with rod length of $300 \mathrm{~nm}$ and B) 3BG5 with rod length of $200 \mathrm{~nm}$. The rod length are colored in red. Near- to mid-IR absorption spectra of the C) 5BG2 and D) 3BG5 dendritic fractals for rod lengths varying from 200 to $400 \mathrm{~nm}$. Arrows in C, D indicates the resonances that were scanned with the s-SNOM.

Microscopic absorbance measurements were first conducted on arrays $\left(50 \times 50 \mu \mathrm{m}^{2}\right)$ of these fractal structures $(10 \times 10$ to $30 \times 30$ individual fractal structures separated by $0.8 \mu \mathrm{m}$ ) using a mid-IR synchrotron source combined with a far-field microscope [16]. As seen in Fig. 1C, the 5BG2 fractal exhibits two dominant resonances in the near- to mid-IR range $\left(6000-1000 \mathrm{~cm}^{-1}\right)$, whereas the 3BG5 fractal has a series of 4-5 resonances that span that same spectral domain (Fig. 1D). These resonances are associated with both dipolar and multipolar modes and their numbers depends on the number of fractal generations. In the first-order generation of the dendritic fractal, there is a single resonance. With each subsequent generation, a new plasmon resonance is introduced [16]. Using a hybridization model of plasmon modes, it appears that the modes with the higher energies are associated with the outermost generation while the modes with the lower energies appears as coupling of modes from the outer structure towards the inner starting dendron [16]. This holds true for the lowest energy resonances of the 3BG5 fractal. Given the spatial extension of its radial geometry with increasing diameter for higher generation structures, the other resonances are also derived from plasmonic coupling between the successive generations as shown using the hybridization model [16]. Fig. 1C, D further demonstrate that by changing the length of the rods (a rod being the building-block unit colored in red in Fig. 1A,B), the spectral positions and the number of resonances can be tailored. The ability to tune the plasmonic properties is a critical factor for applications in optical sensing or when developing an optical filter for multiband spectral ranges. Surface-enhanced infrared absorption is optimum when the infrared resonances of the nanostructured platform match the vibrational modes of interest yielding a Fano interference $[16,18$, 19].

To spatially correlate the plasmon resonances with the structure itself, mid-IR nanoscopy at the scale of a single structure is of crucial interest, especially when calculation is limited due to multiscale converging issues. Furthermore, differences in shape between the modelled structure and the real structures, due to fabrication limitations such as rounding effects for smaller sizes, surface roughness from deposition methods and presence of defects, yields a variety of spectral and spatial differences that are more difficult to all be considered in the EM modelling step. The knowledge of the experimental spatial distributions of the near-field resonances is therefore critical for many applied aspects such as performing differential surface chemistry, polarized measurements or chiral sensing $[8,20,21]$.

The experimental design of the s-SNOM instrument used here (neaSNOM, Neaspec $\mathrm{GmbH}$ ) can be found elsewhere [22]. In these experiments, a tunable quantum cascade laser (QCL) source operating in transmission geometry is focused using a parabolic mirror from below on the fractal surface. The linearly polarized (black arrows in Fig. 2 and 3) source is tuned to the specific plasmon modes corresponding to the resonances indicated in Fig. 1 C, D. On the top of the sample are the AFM tip and a parabolic mirror that is used to collect and guide the scattered light towards the detector. A gold-coated AFM tip (20 nm thick gold layer) was used in these experiments. The coated tip has the two functions of i) enhancing the local field by coupling the plasmon mode of the tip and that of the structure and ii) acting as a local perturber through frustration of the evanescent wave located in the near-field of the sample [15, 23]. The near-field is then converted into far-field and detected with a photodiode using a pseudoheterodyne detection scheme [24]. In this work, the QCLs coupled to the s-SNOM instrument used to excite the plasmon modes had a lower wavelength limit of $\sim 5 \mu \mathrm{m}$ $\left(2000 \mathrm{~cm}^{-1}\right)$.

Five-Branched Structures. An AFM topography was first obtained for a portion of the array containing 5BG2 dendritic fractal entities with rod lengths of $300 \mathrm{~nm}(1.2 \mu \mathrm{m}$ diameter organized in arrays of $27 \times 27$ individual fractals) as shown in Fig. 2 A. The nearfield amplitude $\left(\left|E_{\mathrm{p}}\right|\right)$ map at a set excitation of $\lambda=5.88 \mu \mathrm{m}(1700 \mathrm{~cm}$ ${ }_{1}^{1}$, blue arrow in Fig. 1C) of the scattered light shows the strongest response for the branch oriented along the polarization direction of the excitation (Fig. 2B, black arrow). The corresponding near-field phase $\left(\varphi_{p}\right)$ map and the electric field map (Re(Ep)) of the scattered light exhibits a well-defined $180^{\circ}$ phase change (light blue to light red) along the polarization axis (Fig. 2C,D) indicating a dipolar resonance character $[15,25,26]$. Fig. $2 D$ is the electric field map that corresponds to the real part of the p-polarized scattered fields, $\operatorname{Re}\left(E_{\mathrm{p}}\right)=\left|E_{\mathrm{p}}\right| \cos \varphi_{\mathrm{p}}$. This map can be deduced from the experimentally determined near-field amplitude and phase. Importantly, all the individual fractal structures within the probed area exhibit the same homogeneity in terms of measured amplitude, phase, and real part maps, highlighting the uniform response of the array. Finite-difference time-domain (FDTD) calculations were conducted to examine the polarized character of the scattered near-field. An EM-field calculation was conducted at 
the calculated resonance, which is slightly red-shifted compared to the experimental one $(6.2 \mu \mathrm{m}$ vs. $5.88 \mu \mathrm{m})$. This spectral shift is mainly due to the presence of fabrication defects, dampening from the titanium adhesion layer, and roughness of the gold surface. The calculated normalized $\operatorname{Re}\left(\mathrm{E}_{\mathrm{z}}\right)$ map (Fig. 2E), is nearly identical to the experimental result of Fig. 2D highlighting that, for this particular structure under these specific irradiation conditions, the near-field appears mostly $z$ polarized. FDTD calculations of the amplitude at $\lambda$ $=6.2 \mu \mathrm{m}$ (Fig. $2 \mathrm{~F}$ ) show a distribution comparable to the experimental one. With a large distribution of EM enhancement over the surface of the fractal, this structure is presumably ideal for SEIRA experiments. However, the 5BG2 fractal only exhibits two strong resonances (Fig. 1C), thus providing access to a limited spectral range to explore SEIRA measurements.

To demonstrate the sensitivity of the s-SNOM technique for probing multiresonant structures, where the resonances also have varying spatial distributions, we performed s-SNOM measurements on the 3BG5 fractals that have a higher number of fractal generations and a smaller number of branches.
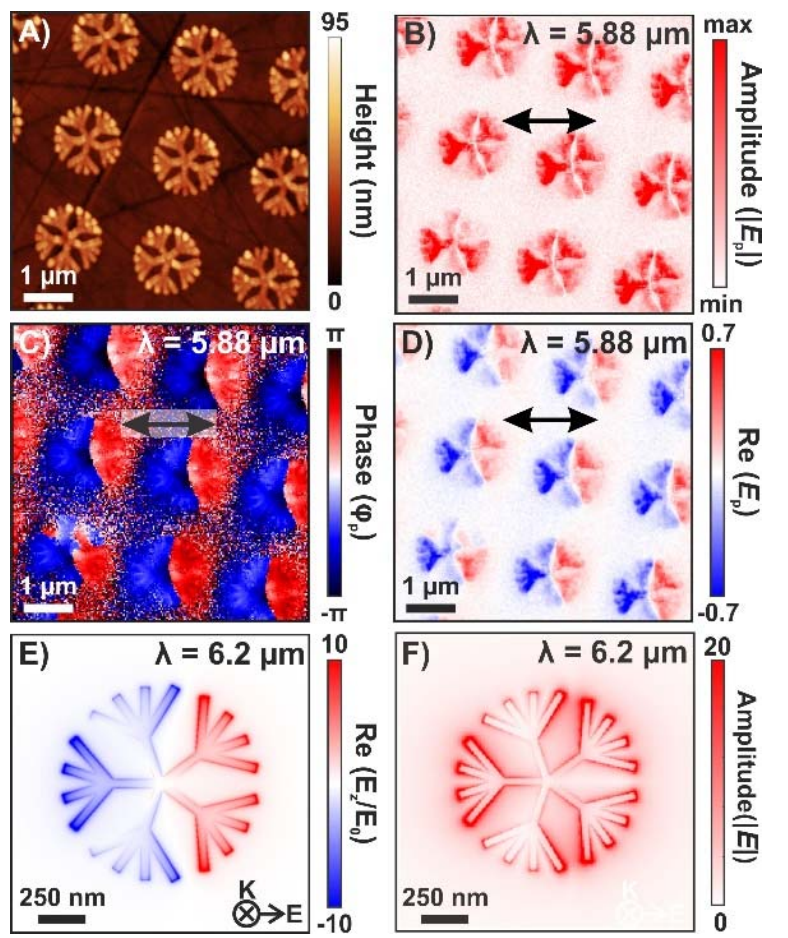

Fig. 2. A) AFM image along with experimental B) amplitude, C) phase, and D) real portion of $E_{\mathrm{p}}$ distribution maps obtained for 5BG2 dendritic fractals with rod lengths of $300 \mathrm{~nm}$ at the low energy resonance. E) Calculated near-field distribution $\left(\operatorname{Re}\left(E_{\mathrm{z}} / E_{0}\right)\right)$, and F) near field amplitude at the calculated resonance.

Three-Branched Structures. 3BG5 fractals with rod length dendrons set to 200 and $350 \mathrm{~nm}$ yielded structures with $2.01 \mu \mathrm{m}$ $(18 \times 18$ array $)$ and $3.50 \mu \mathrm{m}(12 \times 12$ array $)$ diameters respectively, as shown in the AFM images (Fig. 3A-C). For the structure with the $200 \mathrm{~nm}$ rod length, two resonances can be mapped individually at $8.77 \mu \mathrm{m}$ and $6.30 \mu \mathrm{m}$. These resonances correspond to wavenumbers of $1140 \mathrm{~cm}^{-1}$ and $1590 \mathrm{~cm}^{-1}$ in Fig. 1D, respectively. The FDTD resonances are again red-shifted and located at $9.1 \mu \mathrm{m}$ and $6.8 \mu \mathrm{m}$, respectively. For the larger $350 \mathrm{~nm}$ five-branched structure only the mode at $6.13 \mu \mathrm{m}\left(1630 \mathrm{~cm}^{-1}\right)$ can be probed (FDTD resonance at $6.9 \mu \mathrm{m}$ ). Upon excitation with a linearly polarized beam oriented along a branch of the core dendron (black arrows in Fig. 3D-F), the near-field amplitude maps of the scattered light (Fig. 3D-F) show that the brightest regions are homogeneous over the structures independently of the excitation wavelength. Not many differences can be noticed in the amplitude maps for different resonances (Fig. 3D, E) or even for larger structures (Fig. 3F).

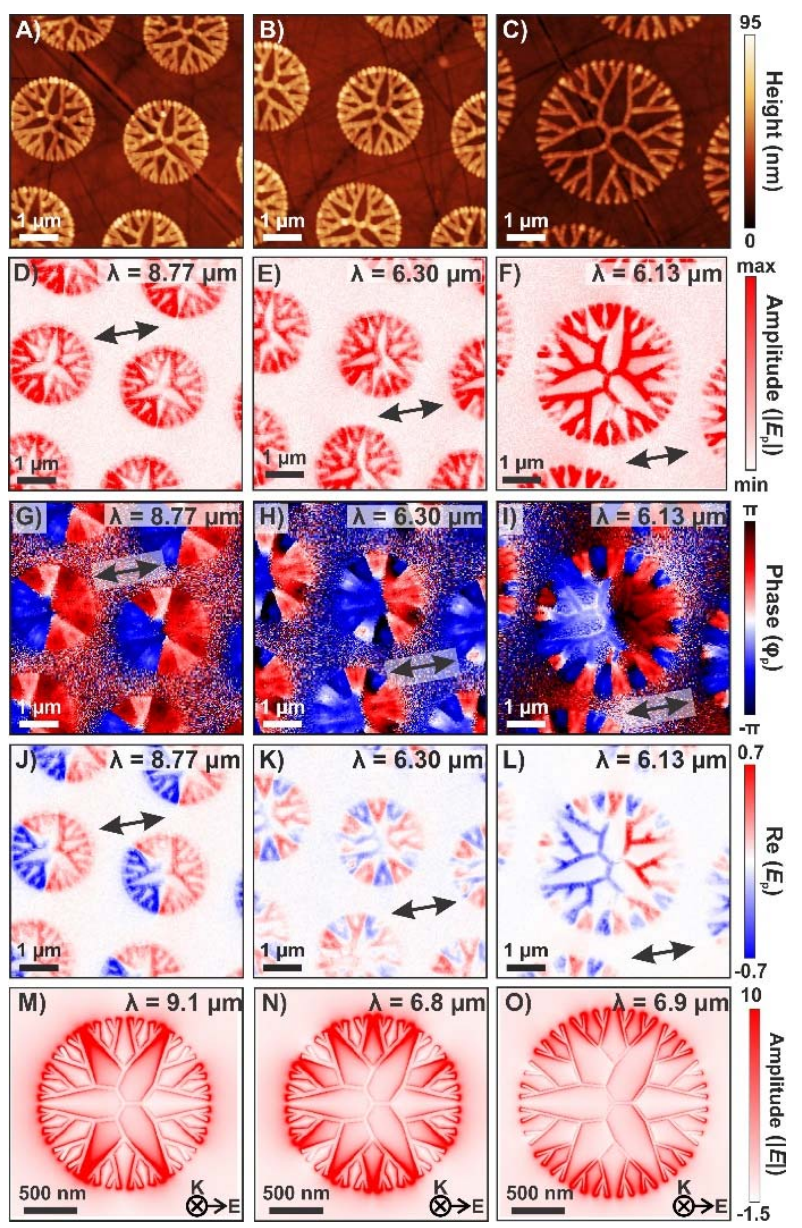

Fig. 3. A-C) AFM images along with experimental D-F) amplitude, G-I) phase, and J-L) real portion of $E_{\mathrm{p}}$ distribution maps obtained for 3BG5 dendritic fractals with rod lengths of $200 \mathrm{~nm}$ (left and middle columns) and $350 \mathrm{~nm}$ (right column) at the indicated wavelengths. M-O) Calculated field amplitude $E=\left(\left|E_{\mathrm{x}}\right|+\left|E_{\mathrm{y}}\right|+\left|E_{\mathrm{z}}\right|\right) / E_{0}$ at the calculated resonance wavelengths.

The amplitude maps show that the plasmon resonance is isotropically distributed over the fractal structure even though the excitation was linearly polarized: the fractal structure radially distributes the plasmon wave over all the sub-units of the fractal. The phase maps reported in Fig. 3G-I are of interest and display peculiar oscillations on the external periphery of the structure. For the lowest observed energy mode $(8.77 \mu \mathrm{m})$ of the $200 \mathrm{~nm}$ structure a phase change of $180^{\circ}$ is observed, with a transition of the phase along the orthogonal direction relative to the input excitation. For the mode located at $6.3 \mu \mathrm{m}$, more phase changes are observed at the outer portion of the structure (Fig. 3H). This effect is even better observed in Fig. 3I, where a total of 22 changes from blue to 
red are observed in the structures from the fourth and fifth-order generations. The calculated amplitude maps shown in Fig. 3M-O considers the amplitude of all three space components, $E_{\mathrm{x}}, E_{\mathrm{y}}, E_{\mathrm{z}}$ with the same weight and resembles the experimental ones in Fig. 3D-F, but the amplitude at the core portion of the fractal does not appear as intense as experimentally measured. These calculations have not been performed with the presence of a gold-coated tip which is also a factor that presumably alters the polarization nature of the local fields. This also highlights also that the infrared maps cannot be considered as the pure metastructure response but rather a convolution of the tip-sample interaction.

The radial distribution of the EM field can then be further examined by looking at the isowavelength map for the three-branched extended structure (Fig. 4A, B)). Here, 54 EM field maps are generated at wavelengths from 1.4 to $12 \mu \mathrm{m}$. For each spatial position, the wavelength corresponding to the resonance with the largest intensity is extracted. The resonance wavelength is then plotted as a function of the spatial coordinates yielding the isowavelength map.
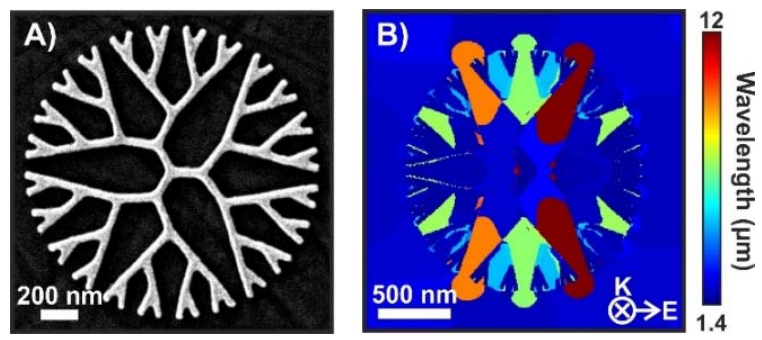

Fig. 4. A) SEM image and B) color-coded isowavelength maps for 3BG5.

At the lowest single energy mapped $(\lambda=9.1 \mu \mathrm{m})$, the largest enhancement extends from the second-order generation to the outermost branches. Athigher energies, the resonances include less of the structure (third- through fifth-order). Additionally, the overlap between the distributions of enhancement is a key factor shown in the isowavelength map. Due to the iterative nature of the fractal resonances, the outermost branches will consistently contribute to enhancement. As such, when performing SEIRA experiments, molecules located in these outer domains will consistently be probed at each resonance wavelength. This contrasts with the typical approach where metasurfaces composed of structures tailored to display a series of narrow wavelength ranges with non-overlapping spatial distributions of EM enhancement are used to achieve enhancement of multiple vibrational modes.

Overall, we have methodically evaluated the plasmonic properties of multiresonant dendritic fractals using both experimental measurements and numerical calculations. By performing s-SNOM measurements, experimental distributions for the near-field enhancement can be obtained at the level of a single structure and compared with numerical results. Overall, highlighting the nature of the scattering field for five- and threebranched structures, and the importance of the tip-sample interaction that can influence the collected near-field maps.

Funding sources and acknowledgments. This research was supported by the Natural Sciences and Engineering Council (NSERC) of Canada. G. Q. W. thanks NSERC for the post-graduate scholarship doctoral. D.M.M. is grateful for an Ontario Graduate Scholarship. Absorption measurements described in this work were performed at the Mid-Infrared (01B1-1) Beamline at the Canadian Light Source. We are grateful to Neaspec GmbH (M. Eisele) for their help regarding the s-NSOM measurements. The authors gratefully acknowledge the Nanofabrication Facility at Western University.

\section{REFERENCES}

1. K. Ueno, T. Oshikiri, Q. Sun, X. Shi, and H. Misawa, Chem. Rev. 118, 2955, (2018).

2. M. Nguyen, I. Kherbouche, S. Gam-Derouich, I. Ragheb, S. Lau-Truong, A. Lamouri, G. Levi, J. Aubard, P. Decorse, N. Félidj, and C. Mangeney, Chem. Commun. 53, 11364, (2017).

3. L. Duempelmann, A. Luu-Dinh, B. Gallinet, and L. Novotny, ACS Photonics 3, 190, (2016).

4. S.-Y. Ding, E.-M. You, Z.-Q. Tian, and M. Moskovits, Chem. Soc. Rev. 46, 4042, (2017).

5. F. Neubrech, C. Huck, K. Weber, A. Pucci, and H. Giessen, Chem. Rev. 117, 5110, (2017).

6. H. Mishra, and C. Geddes, J. Phys. Chem. C 118, 28791, (2014).

7. G. Q. Wallace, S. T. Read, D. M. McRae, S. M. Rosendahl, and F. Lagugné-Labarthet, Adv. Opt. Mater. 6, 1701336, (2018).

8. I. Tijunelyte, I. Kherbouche, S. Gam-Derouich, M. Nguyen, N. LidgiGuigui, M. Lamy de la Chapelle, A. Lamouri, G. Levi, J. Aubard, A. ChevillotBiraud, C. Mangeney, and N. Félidj, Nanoscale Horiz. 3, 53, (2018).

9. G. Q. Wallace, and F. Lagugné-Labarthet, Analyst 144, 13, (2019).

10. X. Wang, M. Li, L. Meng, K. Lin, J. Feng, T. Huang, Z. Yang, and B. Ren, ACS Nano 8, 528, (2014).

11. W. Lum, I. Bruzas, Z. Gorunmez, S. Unser, T. Beck, and L. Sagle, J. Phys. Chem. Lett. 8, 2639, (2017).

12. L. Dong, X. Yang, C. Zhang, B. Cerjan, L. Zhou, M. L. Tseng, Y. Zhang, A. Alabastri, P. Nordlander, and N. J. Halas, Nano Lett. 17, 5768, (2017).

13. R. Yu, L. M. Liz-Marzan, and F. J. Garcia de Abajo, Chem. Soc. Rev. 46, 6710, (2017).

14. M. Schnell, A. Garcia-Etxarri, J. Alkorta, J. Aizpurua, and R. Hillenbrand, Nano Lett. 10, 3524, (2010).

15. T. Neuman, P. Alonso-González, A. Garcia-Etxarri, M. Schnell, R. Hillenbrand, and J. Aizpurua, Laser Photonics Rev. 9, 637, (2015).

16. G. Q. Wallace, H. C. Foy, S. M. Rosendahl, and F. Lagugné-Labarthet, J. Phys. Chem. C 121, 9497, (2017).

17. S. Gottheim, H. Zhang, A. O. Govorov, and N. J. Halas, ACS Nano 9, 3284, (2015).

18. J. Vogt, C. Huck, F. Neubrech, A. Toma, D. Gerbert, and A. Pucci, Phys. Chem. Chem. Phys. 17, 21169, (2015).

19. D. Rodrigo, A. Tittl, A. John-Herpin, O. Limaj, and H. Altug, ACS Photonics 5, 4903, (2018).

20. S. Simoncelli, Y. Li, E. Cortés, and S. A. Maier, ACS Nano 12, 2184, (2018).

21. M. Schnell, P. Sarriugarte, T. Neuman, A. B. Khanikaev, G. Shvets, J. Aizpurua, and R. Hillenbrand, Nano Lett. 16, 663, (2016).

22. V. A. Zenin, A. Andryieuski, R. Malureanu, I. P. Radko, V. S. Volkov, D. K. Gramotnev, A. V. Lavrinenko, and S. I. Bozhevolonyi, Nano Lett. 15, 8148, (2015).

23. R. L. Olmon, P. M. Krenz, A. C. Jones, G. D. Boreman, and M. B. Raschke, Optics Express 16, 20295, (2008).

24. M. Schnell, A. García-Etxarri, A. J. Huber, K. Crozier, J. Aizpurua, and R. Hillenbrand, Nat. Photonics 3, 287, (2009).

25. M. Schnell, A. Garcia-Etxarri, A. J. Huber, K. B. Crozier, A. Borisov, J. Aizpurua, and R. Hillenbrand, J. Phys. Chem. C 114, 7341, (2010).

26. S. E. Grefe, D. Leiva, S. Mastel, S. D. Dhuey, S. Cabrini, P. J. Schuck, and Y. Abate, Phys. Chem. Chem. Phys. 15, 18944, (2013). 


\section{References with Titles}

1. K. Ueno, T. Oshikiri, Q. Sun, X. Shi, and H. Misawa. "Solid-state Plasmonic Solar cells", Chem. Rev. 118, 2955, (2018).

2. M. Nguyen, I. Kherbouche, S. Gam-Derouich, I. Ragheb, S. LauTruong, A. Lamouri, G. Levi, J. Aubard, P. Decorse, N. Félidj, and C. Mangeney. "Regioselective Surface Functionalization of Lithographically Designed Gold Nanorods by Plasmon-Mediated Reduction of Aryl Diazonium Salts", Chem. Commun. 53, 11364, (2017).

3. L. Duempelmann, A. Luu-Dinh, B. Gallinet, and L. Novotny. "Four-Fold Color Filter Based on Plasmonic Phase Retarder", ACS Photonics 3, 190, (2016).

$4 . \quad$ S.-Y. Ding, E.-M. You, Z.-Q. Tian, and M. Moskovits.

"Electromagnetic Theories of Surface-Enhanced Raman Spectroscopy", Chem. Soc. Rev. 46, 4042, (2017).

5. F. Neubrech, C. Huck, K. Weber, A. Pucci, and H. Giessen.

"Surface-Enhanced Infrared Spectroscopy Using Resonant Nanoantennas", Chem. Rev. 117, 5110, (2017).

6. H. Mishra, and C. Geddes. "Metal-Enhanced S1 and Alpha-S1 Fluorescence: Effects of Far-Field Excitation Irradiance on Enhanced Fluorescence", J. Phys. Chem. C 118, 28791, (2014).

7. G. Q. Wallace, S. T. Read, D. M. McRae, S. M. Rosendahl, and F. Lagugné-Labarthet. "Exploiting Anisotropy of Plasmonic Nanostructures with Polarization Modulation Infrared Linear Dichroism Microscopy ( $\mu$ PMIRLD)", Adv. Opt. Mater. 6, 1701336, (2018).

8. I. Tijunelyte, I. Kherbouche, S. Gam-Derouich, M. Nguyen, N. Lidgi-Guigui, M. Lamy de la Chapelle, A. Lamouri, G. Levi, J. Aubard, A. Chevillot-Biraud, C. Mangeney, and N. Félidj. "Multi-Functionalization of Lithographically Designed Gold Nanodisks by Plasmon-Mediated Reduction of Aryl Diazonium Salts", Nanoscale Horiz. 3, 53, (2018).

9. G. Q. Wallace, and F. Lagugné-Labarthet. "Advancements in fractal plasmonics: structures, optical properties, and applications", Analyst 144, 13, (2019).

10. X. Wang, M. Li, L. Meng, K. Lin, J. Feng, T. Huang, Z. Yang, and B. Ren. "Probing the Location of Hot Spots by Surface-Enhanced Raman Spectroscopy: Toward Uniform Substrates", ACS Nano 8, 528, (2014).

11. W. Lum, I. Bruzas, Z. Gorunmez, S. Unser, T. Beck, and L. Sagle.

"Novel Liposome-Based Surface-Enhanced Raman Spectroscopy (SERS) Substrate", J. Phys. Chem. Lett. 8, 2639, (2017).

12. L. Dong, X. Yang, C. Zhang, B. Cerjan, L. Zhou, M. L. Tseng, Y. Zhang, A. Alabastri, P. Nordlander, and N. J. Halas. "Nanogapped Au Antennas for Ultrasensitive Surface-Enhanced Infrared Absorption Spectroscopy", Nano Lett. 17, 5768, (2017).

13. R. Yu, L. M. Liz-Marzan, and F. J. Garcia de Abajo. "Universal Analytical Modeling of Plasmonic Nanoparticles", Chem. Soc. Rev. 46, 6710, (2017).

14. M. Schnell, A. Garcia-Etxarri, J. Alkorta, J. Aizpurua, and R. Hillenbrand. "Phase-Resolved Mapping of the Near-Field Vector and Polarization State in Nanoscale Antenna Gaps", Nano Lett. 10, 3524, (2010).

15. T. Neuman, P. Alonso-González, A. Garcia-Etxarri, M. Schnell, R. Hillenbrand, and J. Aizpurua. "Mapping the Near Fields of Plasmonic Nanoantennas by Scattering-Type Scanning Near-Field Optical Microscopy", Laser Photonics Rev. 9, 637, (2015).

16. G. Q. Wallace, H. C. Foy, S. M. Rosendahl, and F. LagugnéLabarthet. "Dendritic Plasmonics for Mid-Infrared Spectroscopy", J. Phys. Chem. C 121, 9497, (2017).

17. S. Gottheim, H. Zhang, A. O. Govorov, and N. J. Halas. "Fractal Nanoparticle Plasmonics: The Cayley Tree", ACS Nano 9, 3284, (2015).

18. J. Vogt, C. Huck, F. Neubrech, A. Toma, D. Gerbert, and A. Pucci. "Impact of the Plasmonic Near- and Far-Field Resonance-Energy Shift on the Enhancement of Infrared Vibrational Signals", Phys. Chem. Chem. Phys. 17, 21169, (2015).

19. D. Rodrigo, A. Tittl, A. John-Herpin, O. Limaj, and H. Altug. "Selfsimilar multiresonant nanoantenna Arrays for Sensing from Near- to MidInfrared", ACS Photonics 5, 4903, (2018).

20. S. Simoncelli, Y. Li, E. Cortés, and S. A. Maier. "Nanoscale Control of Molecular Self-Assembly Induced by Plasmonic Hot-Electron Dynamics", ACS Nano 12, 2184, (2018).
21. M. Schnell, P. Sarriugarte, T. Neuman, A. B. Khanikaev, G. Shvets, J. Aizpurua, and R. Hillenbrand. "Real-space mapping of the chiral near-field distributions in spiral antennas and planar metasurfaces", Nano Lett. 16, 663, (2016).

22. V. A. Zenin, A. Andryieuski, R. Malureanu, I. P. Radko, V. S. Volkov, D. K. Gramotnev, A. V. Lavrinenko, and S. I. Bozhevolonyi. "Boosting Local Field Enhancement by on-Chip Nanofocusing and Impedance-Matched Plasmonic Antennas", Nano Lett. 15, 8148, (2015).

23. R. L. Olmon, P. M. Krenz, A. C. Jones, G. D. Boreman, and M. B. Raschke. "Near-Field Imaging of Optical Antenna Modes in the MidInfrared", Optics Express 16, 20295, (2008).

24. M. Schnell, A. García-Etxarri, A. J. Huber, K. Crozier, J. Aizpurua, and R. Hillenbrand. "Controlling the Near-Field Oscillations of Loaded Plasmonic Nanoantennas", Nat. Photonics 3, 287, (2009).

25. M. Schnell, A. Garcia-Etxarri, A. J. Huber, K. B. Crozier, A. Borisov, J. Aizpurua, and R. Hillenbrand. "Amplitude- and Phase-Resolved Near-Field Mapping of Infrared Antenna Modes by Transmission-Mode Scattering-Type Near-Field Microscopy", J. Phys. Chem. C 114, 7341, (2010).

26. S. E. Grefe, D. Leiva, S. Mastel, S. D. Dhuey, S. Cabrini, P. J. Schuck, and Y. Abate. "Near-Field Spatial Mapping of Strongly Interacting Multiple Plasmonic Infrared Antennas", Phys. Chem. Chem. Phys. 15, 18944, (2013). 